\title{
Correction to: Next generation technologies
}

Juergen Janek, Philipp Adelhelm

\section{Correction to:}

Chapter 16 in: R. Korthauer (ed.),

Lithium-Ion Batteries: Basics and Applications, https://doi.org/10.1007/978-3-662-53071-9_16

Despite taking great care in the production of our books, errors are not always entirely avoidable. In chapter 16 some author corrections had inadvertently not been incorporated. The chapter has now been corrected and approved by the authors.

The updated online version can be found at https://doi.org/10.1007/978-3-662-53071-9_16 